\title{
Space-Time Block Coding applied to Turbo Coded Multicarrier CDMA
}

\author{
Vincent Le Nir, Maryline Hélard, Rodolphe Le Gouable *
}

\begin{abstract}
In this article, we combine a Space-Time Block Code (STBC) with a Multi-Carrier Code Division Multiplex Access (MC-CDMA) system and a Turbo Code (TC) as channel code. MC-CDMA is likely to be one of the most promising access technique for future wireless communication systems [1][2]. In fact, MC-CDMA exploits the advantages of both the Orthogonal Frequency Division Multiplex (OFDM) multi-carrier modulation and of the Code Division Multiple Access (CDMA) technique. Moreover, the use of a Multiple Input Multiple Output (MIMO) OFDM system associated with a STBC code allows the achievement of both space and frequency diversities [6]. In this paper, we compare several STBC codes such as the Alamouti code [3] and the generalized codes for 3 or 4 antennas proposed by Tarokh [4] applied to a MC-CDMA system leading to different spectral efficiencies. Then, since Turbo Coded MC-CDMA was demonstrated to be very efficient for a Single Input Single Output (SISO) system, allowing the use of a simple Single User (SU) detector [2], we extend this system to Multiple Input Multiple Output (MIMO) systems for different STBC. Simulation results are provided for full load systems.
\end{abstract}

\section{INTRODUCTION}

On one hand, MC-CDMA is a very promising technique for the future wireless communication systems. This technique combines OFDM multi-carrier modulation and CDMA access technique deriving benefits from both techniques notably the high spectral efficiency and the robustness against multipath channels thanks to OFDM and the multi access flexibility and low multiuser inteference provided by CDMA [1][2].

On the other hand, multiple antenna systems were also demonstrated to be a very promising field of research in order to significantly increase the capacity of future wireless communication systems [5]. The introduction of space-time codes [3][4] allowed the spatial diversity to be efficiently exploited. STBC were first proposed by Alamouti [3] in 1998 for $N_{t}=2$ transmit antennas and $N_{r}=1$ receive antenna. Then Tarokh generalized the Alamouti code [4] to a higher number of transmit antennas. The main advantages of STBC are the ability to exploit the transmit diversity and the ease to implement the decoder at the receiver part in terms of complexity. The diversity order of a MIMO system using STBC is the number of transmit antennas times the number of receive antennas $N_{t} N_{r}$.

In this paper, we briefly present a MC-CDMA system in a Single Input Single Output (SISO) antenna system before extending MC-CDMA to a MIMO system including STCB. In addition to Alamouti STBC [3] applied to MC-CDMA system as presented in [6], we detail the combination with the generalized Tarokh [4] codes leading to different spectral efficiency systems. In [2], we demonstrated that a good tradeoff between complexity and BER performance is achieved by using a Single User (SU) detector with a Turbo-Code (TC) for a SISO MC-CDMA transmission. Following this result, we associate a TC as channel coding and a SU detector with our MIMO MC-CDMA scheme.

* France Telecom Research and Development, Rennes, France. E-mail: vincent.lenir@rd.francetelecom.com .
We provide simulation results over an uncorrelated Rayleigh flat fading channels environment for full load systems. In section II we describe a MC-CDMA associated with TC for both SISO and MIMO systems. Thus the STBC codes included in the different tested systems are detailed for both the transmitter and the receiver parts.

\section{MC-CDMA, PRESENTATION}

The MC-CDMA transmission and reception are respectively presented in Figure 1 and Figure 2 in the case of a SISO system as referred in [1][2]. In this section we briefly present the main principles of a MC-CDMA system where data are previously turbo encoded.

First, data input bits are coded by a TC and then converted into symbol vector using adequate mapping and modulation.

The multiuser turbo coded symbol matrix $\mathbf{X}$ including the information of all the users of size $N_{u} \times N$ is denoted:

$\mathbf{X}=\left[\begin{array}{lllll}\mathbf{x}_{1} & \ldots & \mathbf{x}_{\mathbf{n}} & \ldots & \mathbf{x}_{\mathbf{N}}\end{array}\right]$ where $N$ is the number of transmitted symbol vectors and $\mathbf{x}_{\mathbf{n}}=\left[\begin{array}{lllll}x_{1 n} & \ldots & x_{j n} & \ldots & x_{N_{u} n}\end{array}\right]^{T}$ is a vector of length $N_{u}$, where $N_{u}$ is the number of users and $[.]^{T}$ the transpose operation.

The multiuser coded sequence is spread using, for instance, a Fast Hadamard Transform FHT. We consider that the length of the spreading sequences is equal to $L_{c}$. These spreading codes are orthogonal. Here we assume $L_{c} \leq N_{c}$, where $N_{c}$ is the number of subcarriers of the Orthogonal Frequency Division Multiplex (OFDM). Then, an OFDM modulation is applied consisting in the concatenation of a serial to parallel conversion, an Inverse Fast Fourier Transform (IFFT) and an appropriate guard interval depending on the channel impulse response.

We consider frequency non-selective Rayleigh fading per subcarrier. Based on these assumptions and considering ideal time and frequency interleaving, the independent complex channel fading coefficients are considered uncorrelated for each subcarrier $k$. Hence, the theoretical channel response, for the $k$ th subcarrier can be estimated by $h_{k}=\rho_{k} e^{i \theta_{k}}$. This modelling has the advantage of giving the asymptotical performance of the system since optimal frequency diversity is obtained.

In the SISO case, the signal received for the $L_{c}$ subcarriers is equal to:

$$
\mathbf{R}=\mathbf{H C X}+\mathbf{N}
$$

where $\mathbf{R}=\left[\begin{array}{lllll}\mathbf{r}_{\mathbf{1}} & \ldots & \mathbf{r}_{\mathbf{n}} \ldots \mathbf{r}_{\mathbf{N}}\end{array}\right]$ is a matrix of size $L_{c} \times N$, with $\mathbf{r}_{\mathbf{n}}=\left[\begin{array}{lllll}r_{1 n} & \ldots & r_{k n} & \ldots & r_{L_{c} n}\end{array}\right]^{T}$ a vector of length $L_{c}$.

$\mathbf{H}$ is a $L_{c} \times L_{c}$ time varying diagonal matrix, each element of the diagonal standing for the frequency channel response of each subcarrier, 


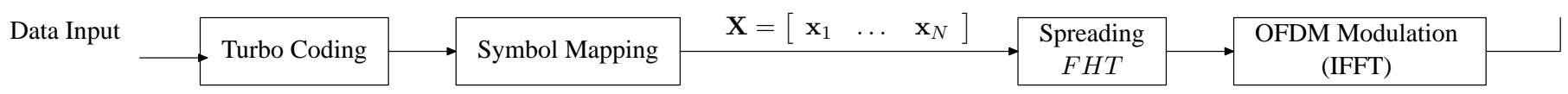

Fig. 1. MC-CDMA SISO Transmitter

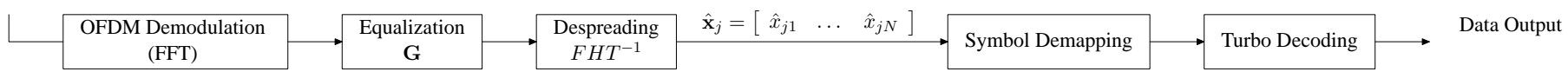

Fig. 2. MC-CDMA SISO Receiver

$\mathbf{C}=\left[\begin{array}{lllll}\mathbf{c}_{\mathbf{1}} & \ldots & \mathbf{c}_{\mathbf{j}} & \ldots & \mathbf{c}_{\mathbf{N}_{\mathbf{u}}}\end{array}\right]$ is the $L_{c} \times N_{u}$ matrix of user's spreading codes,

$\mathbf{c}_{\mathbf{j}}=\left[\begin{array}{lllll}c_{1 j} & \ldots & c_{k j} & \ldots & c_{L_{c} j}\end{array}\right]^{T}$ is the vector of length $L_{c}$ of user $j$,

and $\mathbf{N}$ is the Additive White Gaussian Noise (AWGN) matrix of size $L_{c} \times N$.

In this paper, we only consider a Single User (SU) MMSE detector. In fact, for a SISO transmission and Turbo Coded MCCDMA, a SU MMSE detector was demonstrated to offer very good performance, avoiding the use of more complex Multiple Users (MU) detectors [2]. Hence, at the receiver in case of SU detector, an equalizer $g_{k}$ corrects the $L_{c}$ amplitude and phase variations of each subcarrier $k . \mathbf{G}$ is a diagonal matrix containing the equalization coefficients $g_{k}$. We can consider that $\mathbf{G}=\tilde{\mathbf{H}}^{\dagger}=\tilde{\mathbf{H}}^{*}$, where $[.]^{\dagger}$ and [.] ${ }^{*}$ respectively denote the transpose conjugate and the conjugate operations and $\tilde{\mathbf{H}}^{*}$ the conjugate diagonal matrix of the normalized channel coefficient for each subcarrier. Thus, the equalization coefficient can be written as follows:

$$
g_{k}=\tilde{h}_{k}^{*}=\frac{h_{k}^{*}}{\left|h_{k}\right|^{2}+\frac{1}{\gamma_{k}}}
$$

with $\gamma_{k}$ the Signal to Noise Ratio (SNR) for the subcarrier k. After equalization, the resultant signal is:

$$
\mathbf{Y}=\mathbf{G H C X}+\mathbf{G N}
$$

where $\mathbf{Y}=\left[\mathbf{y}_{\mathbf{1}} \ldots \mathbf{y}_{\mathbf{n}} \ldots \mathbf{y}_{\mathbf{N}}\right]$ is the $L_{c} \times N$ matrix of the equalized signals, with $\mathbf{y}_{n}=\left[\begin{array}{lllll}y_{1 n} & \ldots & y_{k n} & \ldots & y_{L_{c} n}\end{array}\right]^{T}$ the $L_{c}$ length vector of the received symbol $n$.

The final step consists in executing the despreading by applying the IFHT to the vector $\mathbf{Y}$ in order to detect the $N$ coded symbols $x_{j n}$ transmitted by the user $j$. Finally soft demapping and turbo decoding is processed.

\section{COMBINING MC-CDMA AND STBC}

The MIMO MC-CDMA transmission and reception are respectively presented in Figure 3 and Figure 4.

\section{A. Transmitter Part}

In the case of $N_{t}=2,3$ or 4 transmit antennas, the following STBC $\mathcal{A}=\mathcal{G}_{2}, \mathcal{G}_{3}, \mathcal{G}_{4}, \mathcal{H}_{3}, \mathcal{H}_{4}$ as defined by [4] are used in this paper. They are combined with either BPSK, QPSK, 8PSK or 16QAM constellation in order to compare systems at the same spectral efficiencies of 1,2 , or $3 \mathrm{bps} / \mathrm{Hz}$.

In the multiuser case, the coded sequences are defined by:

$$
\begin{aligned}
& \mathcal{G}_{2}^{\mathrm{X}}=\left[\begin{array}{rr}
\mathbf{x}_{1} & -\mathbf{x}_{2}^{*} \\
\mathbf{x}_{2} & \mathbf{x}_{1}^{*}
\end{array}\right] \\
& \mathcal{G}_{3}^{\mathbf{X}}=\left[\begin{array}{rrrrrrrr}
\mathbf{x}_{1} & -\mathbf{x}_{2} & -\mathbf{x}_{3} & -\mathbf{x}_{4} & \mathbf{x}_{1}^{*} & -\mathbf{x}_{2}^{*} & -\mathbf{x}_{3}^{*} & -\mathbf{x}_{4}^{*} \\
\mathbf{x}_{2} & \mathbf{x}_{1} & \mathbf{x}_{4} & -\mathbf{x}_{3} & \mathbf{x}_{2}^{*} & \mathbf{x}_{1}^{*} & \mathbf{x}_{4}^{*} & -\mathbf{x}_{3}^{*} \\
\mathbf{x}_{3} & -\mathbf{x}_{4} & \mathbf{x}_{1} & \mathbf{x}_{2} & \mathbf{x}_{3}^{*} & -\mathbf{x}_{4}^{*} & \mathbf{x}_{1}^{*} & \mathbf{x}_{2}^{*}
\end{array}\right] \\
& \mathcal{G}_{4}^{\mathbf{X}}=\left[\begin{array}{rrrrrrrr}
\mathbf{x}_{1} & -\mathbf{x}_{2} & -\mathbf{x}_{3} & -\mathbf{x}_{4} & \mathbf{x}_{1}^{*} & -\mathbf{x}_{2}^{*} & -\mathbf{x}_{3}^{*} & -\mathbf{x}_{4}^{*} \\
\mathbf{x}_{2} & \mathbf{x}_{1} & \mathbf{x}_{4} & -\mathbf{x}_{3} & \mathbf{x}_{2}^{*} & \mathbf{x}_{1}^{*} & \mathbf{x}_{4}^{*} & -\mathbf{x}_{3}^{*} \\
\mathbf{x}_{3} & -\mathbf{x}_{4} & \mathbf{x}_{1} & \mathbf{x}_{2} & \mathbf{x}_{3}^{*} & -\mathbf{x}_{4}^{*} & \mathbf{x}_{1}^{*} & \mathbf{x}_{2}^{*} \\
\mathbf{x}_{4} & \mathbf{x}_{3} & -\mathbf{x}_{2} & \mathbf{x}_{1} & \mathbf{x}_{4}^{*} & \mathbf{x}_{3}^{*} & -\mathbf{x}_{2}^{*} & \mathbf{x}_{1}^{*}
\end{array}\right] \\
& \mathcal{H}_{3}^{\mathbf{X}}=\left[\begin{array}{cccc}
\mathbf{x}_{1} & -\mathbf{x}_{\mathbf{2}}^{*} & \frac{\mathbf{x}_{3}^{*}}{\sqrt{2}} & \frac{\mathbf{x}_{3}^{*}}{\sqrt{2}} \\
\mathbf{x}_{2} & \mathbf{x}_{1}^{*} & \frac{\mathbf{x}_{3}^{*}}{\sqrt{2}} & -\frac{\mathbf{x}_{3}^{*}}{\sqrt{2}} \\
\frac{\mathbf{x}_{3}}{\sqrt{2}} & \frac{\mathbf{x}_{3}}{\sqrt{2}} & \frac{\left(-\mathbf{x}_{1}-\mathbf{x}_{1}^{*}+\mathbf{x}_{2}-\mathbf{x}_{2}^{*}\right)}{\sqrt{2}} & \frac{\left(-\mathbf{x}_{2}-\mathbf{x}_{2}^{*}+\mathbf{x}_{1}-\mathbf{x}_{1}^{*}\right)}{\sqrt{2}}
\end{array}\right] \\
& \mathcal{H}_{4}^{\mathbf{X}}=\left[\begin{array}{cccc}
\mathbf{x}_{1} & -\mathbf{x}_{2}^{*} & \frac{\mathbf{x}_{3}^{*}}{\sqrt{2}} & \frac{\mathbf{x}_{3}^{*}}{\sqrt{2}} \\
\mathbf{x}_{2} & \mathbf{x}_{1}^{*} & \frac{\mathbf{x}_{3}^{*}}{\sqrt{2}} & -\frac{\mathbf{x}_{3}^{*}}{\sqrt{2}} \\
\frac{\mathbf{x}_{3}}{\sqrt{2}} & \frac{\mathbf{x}_{3}}{\sqrt{2}} & \frac{\left(-\mathbf{x}_{1}-\mathbf{x}_{1}^{*}+\mathbf{x}_{2}-\mathbf{x}_{2}^{*}\right)}{\sqrt{2}} & \frac{\left(-\mathbf{x}_{2}-\mathbf{x}_{2}^{*}+\mathbf{x}_{1}-\mathbf{x}_{1}^{*}\right)}{\sqrt{2}} \\
\frac{\mathbf{x}_{3}}{\sqrt{2}} & -\frac{\mathbf{x}_{3}}{\sqrt{2}} & \frac{\left(\mathbf{x}_{2}+\mathbf{x}_{2}^{*}+\mathbf{x}_{1}-\mathbf{x}_{1}^{*}\right)}{\sqrt{2}} & -\frac{\left(\mathbf{x}_{1}+\mathbf{x}_{1}^{*}+\mathbf{x}_{2}-\mathbf{x}_{2}^{*}\right)}{\sqrt{2}}
\end{array}\right]
\end{aligned}
$$

where $\mathbf{X}$ is the multiuser turbo coded symbol matrix of size $N_{u} \times N$ including the information of all the users.

Since $L$ time slots are used to transmit $N$ symbols, the rate $R$ of the STBC code is usually defined by $R=N / L$. Hence, for $\mathcal{G}_{2} \mathrm{R}=1$, for $\mathcal{G}_{3}$ and $\mathcal{G}_{4} \mathrm{R}=1 / 2$, and for $\mathcal{H}_{3}$ and $\mathcal{H}_{4} \mathrm{R}=3 / 4$.

\section{B. Receiver part}

After STBC coding, the multiuser coded sequence $\mathcal{A}^{\mathrm{X}}$ is spread using, for instance, a FHT over each STBC coded symbol as with classical MC-CDMA. Then, the Multicarrier modulation is easily performed by an IFFT. We can note that, at the transmitter, the FHT could have been performed before STBC scheme with only a penalty in terms of complexity.

The STBC are carried out on several symbols that are efficiently recombined at the receiver if the channel is assumed to be time invariant over these symbols. For the Alamouti code (rate $R=1$ ) and the Tarokh codes $\left(R=\frac{1}{2}, R=\frac{3}{4}\right)$ applied to MC-CDMA, this assumption is required over respectively $L=2,8$ and 4 OFDM symbols. Then, for this study, frequency non-selective Rayleigh fading per subcarrier and time invariance during $L$ symbols are assumed as well as perfect channel estimation. Moreover, we consider uncorrelated channels from each transmit antenna $t$ to each receive antenna $r$. Under these assumptions and considering ideal time and frequency interleaving the complex channel fading coefficients are considered independent for each subcarrier $k$. Hence, the theoretical channel response, for the $k^{\text {th }}$ subcarrier, from transmit antenna $t$ to receive antenna $r$ can be estimated by $h_{t r, k}=\rho_{t r, k} e^{i \theta_{t r, k}}$. This mod- 


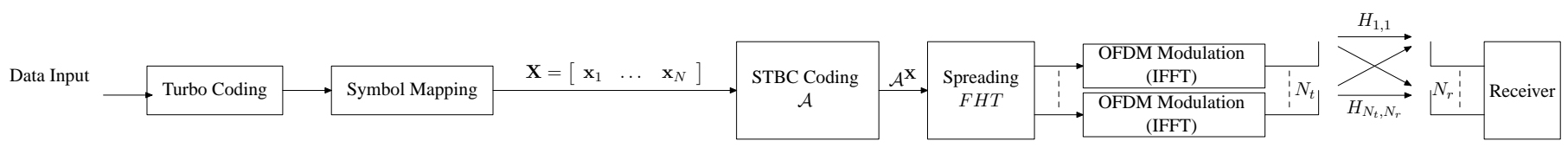

Fig. 3. MC-CDMA MIMO Transmitter

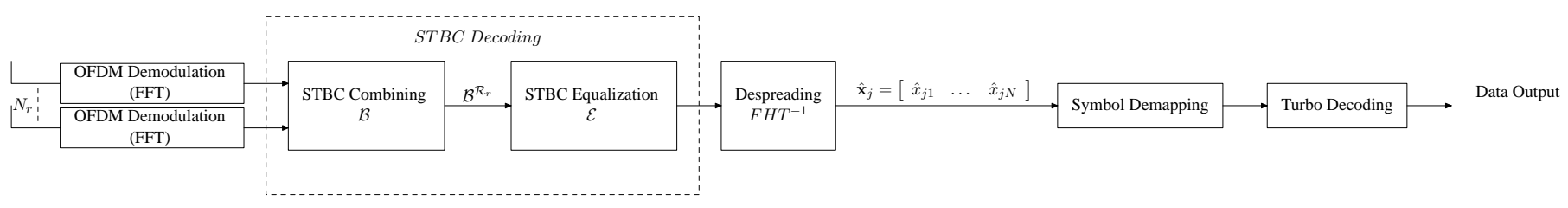

Fig. 4. MC-CDMA MIMO Receiver

elling has the advantage of giving the asymptotical performance of the system, since optimal spatial and frequency diversity is obtained. In the MIMO case, when STBC is used, the signal received during $L$ adjacent OFDM symbols is equal to:

$$
\mathcal{R}_{r}=\mathcal{J D}_{r} \mathcal{C} \mathcal{A}^{\mathbf{X}}+\mathcal{N}_{r}
$$

where $\mathcal{R}_{r}=\left[\mathbf{r}_{1 r} \ldots \mathbf{r}_{l r} \ldots \mathbf{r}_{L r}\right]$ is a $L_{c} \times L$ matrix of the $L$ received signals $\mathbf{r}_{l r}$ at the $r^{\text {th }}$ antenna, with $\mathbf{r}_{l r}$ the vector of the $L_{c}$ subcarriers received at time $l, \mathcal{J}=\mathbf{1}_{1 \times N_{t}} \otimes \mathbf{I}_{L_{c}}$ is a matrix of size $L_{c} \times N_{t} L_{c}$ corresponding to the summation of the signals,

$$
\mathcal{D}_{r}=\left[\begin{array}{ccccc}
\mathbf{H}_{1 \mathbf{r}} & 0 & \ldots & \ldots & 0 \\
0 & \ddots & \ddots & & \vdots \\
\vdots & \ddots & \mathbf{H}_{\mathbf{t r}} & \ddots & \vdots \\
\vdots & & \ddots & \ddots & 0 \\
0 & \ldots & \ldots & 0 & \mathbf{H}_{\mathbf{N}_{\mathbf{t}} \mathbf{r}}
\end{array}\right] \text { is the diagonal channel }
$$

matrix of size $N_{t} L_{c} \times N_{t} L_{c}$ where $\mathbf{H}_{t r}$ is a $L_{c} \times L_{c}$ diagonal matrix with $h_{t r, k}$ the $k^{t h}$ element, $\mathcal{C}=\mathbf{I}_{N_{t}} \otimes \mathbf{C}$ of size $N_{t} L_{c} \times N_{t} N_{u}, \mathcal{A}^{\mathbf{X}}$ is the $N_{t} N_{u} \times L$ matrix of multiuser coded sequences, $\mathcal{N}_{r}$ is $L_{c} \times L$ matrix of the noise vector $\mathbf{n}_{l r}$ of length $L$, with $\mathbf{n}_{l r}$ the vector of the $L_{c}$ noise terms at time $l$. The STBC decoding and equalization, detailed in the next part, are then performed before despreading and Turbo decoding.

\section{STBC Decoding and MC-CDMA Equalization}

The first step of STBC decoding consists in applying to the received matrix $\mathcal{R}_{r}$ an appropriate combining related to the STBC scheme $\mathcal{A}$ used at the transmitter in order to obtain the column vector $\mathcal{B}^{\mathcal{R}_{r}}$. For instance, with $\mathcal{A}=\mathcal{G}_{2}$, we have:

$$
\mathcal{B}^{\mathcal{R}_{r}}=\left[\begin{array}{r}
\mathbf{r}_{1 r} \\
-\mathbf{r}_{2 r}^{*}
\end{array}\right]
$$

With $\mathcal{A}=\mathcal{G}_{3}$ or $\mathcal{A}=\mathcal{G}_{4}$, we have:

$$
\mathcal{B}^{\mathcal{R}_{r}}=\left[\begin{array}{c}
\mathbf{r}_{1 r} \\
-\mathbf{r}_{2 r} \\
-\mathbf{r}_{3 r} \\
-\mathbf{r}_{4 r} \\
\mathbf{r}_{5 r}^{*} \\
-\mathbf{r}_{6 r}^{*} \\
-\mathbf{r}_{7 r}^{*} \\
-\mathbf{r}_{8 r}^{*}
\end{array}\right]
$$

We can note that for these three codes, the first decoding process consists in applying the first row of the STBC applied at the transmitter. With and $\mathcal{A}=\mathcal{H}_{3}$ or $\mathcal{A}=\mathcal{H}_{4}$, we have:

$$
\mathcal{B}^{\mathcal{R}_{r}}=\left[\begin{array}{c}
\mathbf{r}_{1 r} \\
\mathbf{r}_{2 r} \\
\mathbf{r}_{2 r}^{*} \\
\mathbf{r}_{3 r} \\
\mathbf{r}_{3 r}^{*} \\
\mathbf{r}_{4 r} \\
\mathbf{r}_{4 r}^{*}
\end{array}\right]
$$

This process should be performed on each receive antenna $r=$ $1 \ldots N_{r}$.

During the second step, an equalization matrix $\mathcal{E}^{\mathbf{G}_{r}}$ is obtained for each receive antenna by applying to the equalization coefficients matrices $\mathbf{G}_{t r}$ an appropriate mapping related to the STBC scheme $\mathcal{A}$ used at the transmitter. $\mathbf{G}_{t r}$ is a diagonal matrix containing the SU equalization coefficients $g_{t r, k}$, for the channel $\operatorname{tr}(t \in\{1,2,3,4\}, r \in\{1,2\})$.

We can consider that $\mathbf{G}_{t r}=\tilde{\mathbf{H}}_{t r}^{\dagger}=\tilde{\mathbf{H}}_{t r}^{*}$, where $\tilde{\mathbf{H}}_{t r}^{*}$ is the conjugate diagonal matrix of the normalized channel coefficient for each of the $L_{c}$ subcarriers. Thus, the equalization coefficient can be written:

$$
g_{t r, k}=\tilde{h}_{t r, k}^{*}=\frac{h_{t r, k}^{*}}{\sum_{t=1}^{N_{t}} \sum_{r=1}^{N_{r}}\left|h_{t r, k}\right|^{2}+\frac{1}{\gamma_{r, k}}}
$$

with $\gamma_{r, k}$ the Signal to Noise Ratio (SNR) for the subcarrier $\mathrm{k}$ and the receive antenna $r$.

When $\mathcal{G}_{2}$ or $\mathcal{G}_{4}$ is used, we applied directly the STBC scheme to the equalization matrices $\mathcal{E}^{\mathbf{G}_{r}}=\mathcal{G}_{2}{ }^{\mathbf{G}_{r}}$ or $\mathcal{E}^{\mathbf{G}_{r}}=\mathcal{G}_{4} \mathbf{G}_{r}$.

When $\mathcal{G}_{3}$ is used (4 symbols transmitted from 3 antennas), we cannot directly apply $\mathcal{G}_{3}$ on the equalization coefficients matrices $\mathbf{G}_{t r}$ but the folowing one:

$$
\mathcal{E}^{\mathbf{G}_{r}}=\left[\begin{array}{cccccccc}
\mathbf{G}_{1 r}-\mathbf{G}_{2 r} & -\mathbf{G}_{3 r} & 0 & \mathbf{G}_{1 r}^{*} & -\mathbf{G}_{2 r}^{*} & -\mathbf{G}_{3 r}^{*} & 0 \\
\mathbf{G}_{2 r} & \mathbf{G}_{1 r} & 0 & -\mathbf{G}_{3 r} & \mathbf{G}_{2 r}^{*} & \mathbf{G}_{1 r}^{*} & 0 & -\mathbf{G}_{3 r}^{*} \\
\mathbf{G}_{3 r} & 0 & \mathbf{G}_{1 r} & \mathbf{G}_{2 r} & \mathbf{G}_{3 r}^{*} & 0 & \mathbf{G}_{1 r}^{*} & \mathbf{G}_{2 r}^{*} \\
0 & \mathbf{G}_{3 r} & -\mathbf{G}_{2 r} & \mathbf{G}_{1 r} & 0 & \mathbf{G}_{3 r}^{*} & -\mathbf{G}_{2 r}^{*} & \mathbf{G}_{1 r}^{*}
\end{array}\right]
$$

In this case, when the symbol $\mathbf{x}_{n}$ was not transmitted at time slot $l, 0$ is present at the $n^{t h}$ row and $l^{t h}$ column of $\mathcal{E}^{\mathbf{G}_{r}}$. 
In the same way, when $\mathcal{H}_{3}$ or $\mathcal{H}_{4}$ is used at the transmitter, we respectively apply:

$$
\begin{aligned}
& \mathcal{E}^{\mathbf{G}_{r}}=\left[\begin{array}{ccccccc}
\mathbf{G}_{1 r} & 0 & \mathbf{G}_{2 r}^{*} & -\frac{\mathbf{G}_{3 r}}{2} & -\frac{\mathbf{G}_{3 r}^{*}}{2} & \frac{\mathbf{G}_{3 r}}{2} & -\frac{\mathbf{G}_{3 r}^{*}}{2} \\
\mathbf{G}_{2 r} & 0 & -\mathbf{G}_{1 r}^{*} & \frac{\mathbf{G}_{3 r}}{2} & -\frac{\mathbf{G}_{3 r}^{*}}{2} & \frac{\mathbf{G}_{3 r}}{2} & \frac{\mathbf{G}_{3 r}^{*}}{2} \\
\frac{\mathbf{G}_{3 r}}{\sqrt{2}} & \frac{\mathbf{G}_{3 r}}{\sqrt{2}} & 0 & 0 & \frac{\mathbf{G}_{1 r}^{*}+\mathbf{G}_{2 r}^{*}}{\sqrt{2}} & 0 & \frac{\mathbf{G}_{1 r}^{*}-\mathbf{G}_{2 r}^{*}}{\sqrt{2}}
\end{array}\right]
\end{aligned}
$$

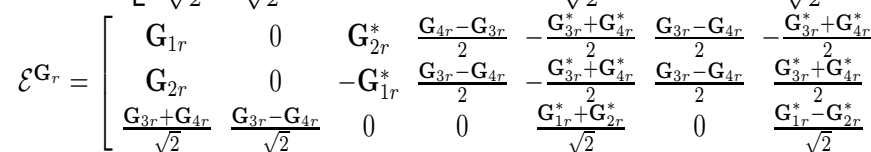

The next step consists in performing the equalization process for each receive antenna $r$.

Thus, to recover the $N$ vectors $\mathbf{y}_{n}$ of length $N_{u}, \mathcal{E}^{\mathbf{G}_{r}}$ is multiplied by $\mathcal{B}^{\mathcal{R}_{r}}$ in order to equalize the received signals and to combine them. Finally, the signals resulting from the $N_{r}$ receive antennas are the simple addition of the signals combined from each antenna. After equalization and combination, the received signal is equal to:

$$
\begin{gathered}
\mathcal{Y}=\left[\begin{array}{c}
\mathbf{y}_{1} \\
\vdots \\
\mathbf{y}_{N}
\end{array}\right] \\
\mathcal{Y}=\sum_{r=1}^{N_{r}} \mathcal{E}^{\mathbf{G}_{r}} \mathcal{B}^{\mathcal{R}_{r}}
\end{gathered}
$$

For instance, with a 2 antenna STBC, we have:

$$
\left[\begin{array}{l}
\mathbf{y}_{1} \\
\mathbf{y}_{2}
\end{array}\right]=\sum_{r=1}^{N_{r}}\left[\begin{array}{l}
\mathbf{G}_{1 r} \mathbf{r}_{1 r}+\mathbf{G}_{2 r}^{*} \mathbf{r}_{2 r}^{*} \\
\mathbf{G}_{2 r} \mathbf{r}_{1 r}-\mathbf{G}_{1 r}^{*} \mathbf{r}_{2 r}^{*}
\end{array}\right]
$$

For the $k^{t h}$ subcarrier, we can write:

$$
\left[\begin{array}{l}
y_{1, k} \\
y_{2, k}
\end{array}\right]=\sum_{r=1}^{N_{r}}\left[\begin{array}{l}
g_{1 r, k} r_{1 r, k}+g_{2 r, k}^{*} r_{2 r, k}^{*} \\
g_{2 r, k} r_{1 r, k}-g_{1 r, k}^{*} r_{2 r, k}^{*}
\end{array}\right]
$$

The final step consists in executing the despreading by applying the inverse FHT to the vector $\mathcal{Y}$ in order to detect the $N$ symbols $x_{j, n}$ transmitted by the user $j$. Finally, a soft demapping is processed in order to feed the turbo decoder which executes the channel decoding over several iterations.

\section{Simulation Results}

We study the performance of the Alamouti and the 4 Tarokh STBC codes for respectively $N_{t}=2,3$ or 4 transmit antennas adapted to MC-CDMA transmission scheme. We assume that the receiver has a perfect knowledge of the MIMO channel, where each subcarrier follows an independent flat Rayleigh fading for each active user and for each MIMO subchannel. The assumption of a full load system is made, i.e. the number of active users $N_{u}$ is equal to the length of the spreading WalshHadamard code $N_{c}=64$. In our simulations, the transmit or the receive signal powers are equal for any number of transmit and receive antennas in order to compare the performance of the five STBC with the same power.

In the Figures 5, 6, 7 we compare systems with $N_{t}=2,3$ or 4 transmit antennas and $N_{r}=1$ or 2 receive antennas without TC for spectral efficiencies of $\eta=1,2$ or $3 \mathrm{bps} / \mathrm{Hz}$ with a MMSE SU detector at full load. For $\eta=1$, the STBC are combined with either BPSK or QPSK constellation both leading to same BER performance over a gaussian SISO channel. The best BER performance is consequently obtained for the STBC offering the better spatial diversity, thus in our study with $N_{t} \cdot N_{r}=4$ corresponding to the $\mathrm{G} 2 \mathrm{x} 2$ or $\mathrm{G} 4 \mathrm{x} 1$ systems. For $\eta=2$, the best BER results are obtained by the Alamouti code associated with QPSK. In fact, Tarokh codes leads to worse performance mainly due to their association with 16QAM which is less robust than QPSK. For $\eta=3$, as the performance of gaussian SISO sytems with 8PSK and 16QAM are closer, there is less difference between Tarokh codes associated with 16QAM and 8PSK associated with G2x1. Nevertheless, Alamouti G2x2 system remains the best one.

The second set of Figures 8, 9, 10 shows the BER performance of the same systems including a duo-binary Convolutional Turbo Code [7] of rate $\frac{1}{2}$. Thus, we compare our systems for specral efficiencies of $\eta=0.5,1$ or $1.5 \mathrm{bps} / \mathrm{Hz}$ with a MMSE detector. We use this turbo code in a packet mode and we carry out 6 iterations of decoding. For each spectral efficiency, the order of BER performance curves are the same than the order obtained at a low SNR coresponding approximately to a Bit Error Rate of $6.10^{-2}$ before Turbo Decoding.

To sum up, without TC, a MIMO MC-CDMA system at full load provides quite similar results to those obtained with a single carrier modulation [4]. As previously demonstrated for SISO MC-CDMA system [2], the BER performance with TC mainly depends on performance at low SNR without TC, we obtain the same order for the curves of the different systems. Moreover, the MIMO G2x2 system applying Alamouti STBC for a multiple antenna system with $N_{t}=2$ transmit antennas and $N_{r}=2$ receive antennas always outperforms the other tested systems whatever the use of $\mathrm{TC}$ thanks to its $\mathrm{R}=1$ rate and its space diversity.

\section{CONCLUSION}

The space diversity is efficiently exploited thanks to a MIMO system combined with of MC-CDMA over a frequency selective Rayleigh channel. As for SISO system, when using TC, the best BER performance are achieved by the system which gives the best BER performance at low SNR without TC. Nevertheless, STBC applied to Turbo Coded MC-CDMA offers a very good tradeoff between performance and complexity with a MMSE SU detector especially when using Alamouti STBC G2x2 system with 2 transmit and 2 receive antennas. These results obtained over a theoretical perfectly estimated channel should further be completed by simulations over more realistic MIMO channels. Further studies would also include channel estimation and results will be provided for other load systems as part of MATRICE projet [8].

\section{ACKNOWLEDGEMENT}

The work presented in this paper was supported by the European IST-2001-32620 MATRICE project (MC-CDMA Transmission Techniques for Integrated Broadband Cellular Systems) [8]. 


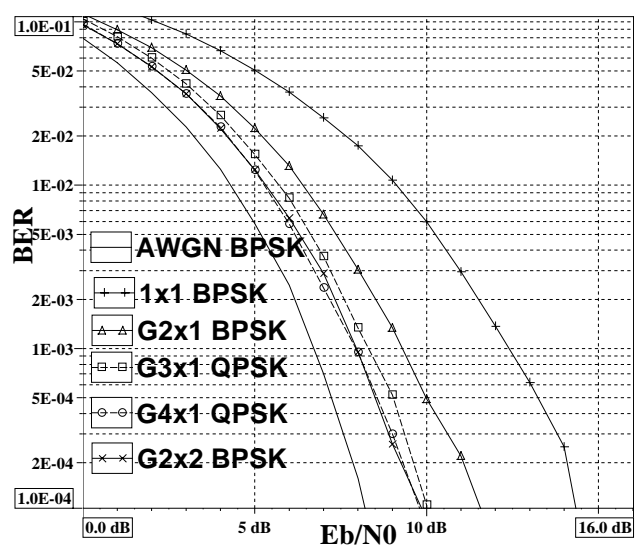

Fig. 5. Comparison of $\eta=1 \mathrm{bps} / \mathrm{Hz}$ systems without channel coding

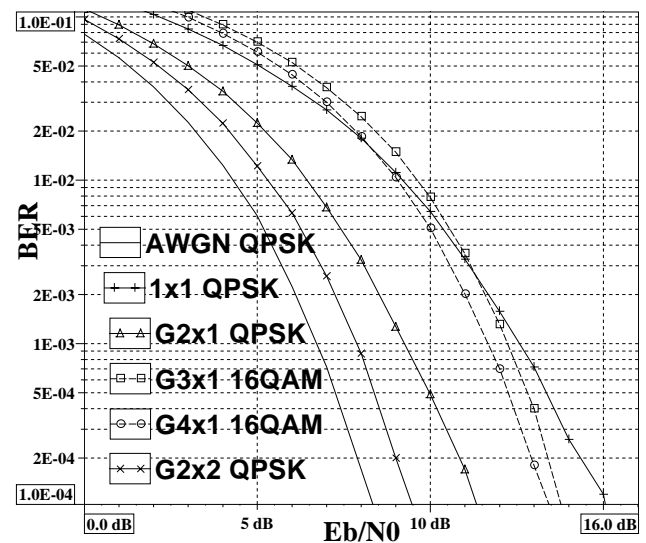

Fig. 6. Comparison of $\eta=2 \mathrm{bps} / \mathrm{Hz}$ systems without channel coding

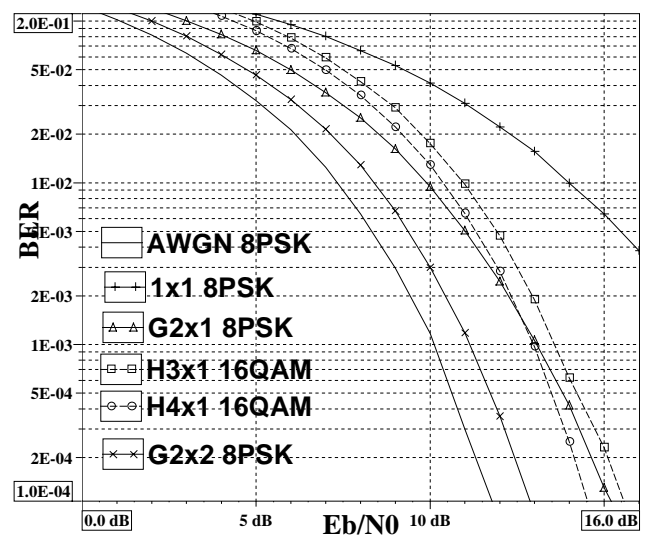

Fig. 7. Comparison of $\eta=3 \mathrm{bps} / \mathrm{Hz}$ systems without channel coding

\section{REFERENCES}

[1] N. Yee, J. P. Linnartz, G. Fettweis, Multicarrier CDMA in Indoor Wireless Radio Networks, IEEE PIMRC'93, pp. 109-113, Yokohama, Japan, 1993.

[2] M. Hélard, R. Le Gouable, J.-F. Hélard, J.-Y Baudais, Multicarrier CDMA Techniques for Future Wideband Wireless Networks, Annales des Telecoms, Special issue on UMTS, May 2001.

[3] S. M. Alamouti, A Simple Transmit Diversity Technique for Wireless Communications, IEEE Journal on Selected Areas in Communications, Vol. 16, No. 8, October 1998, pp. 1451-1458.

[4] V. Tarokh, H. Jafarkhani, A. R. Calderbank, Space-Time Block Codes from

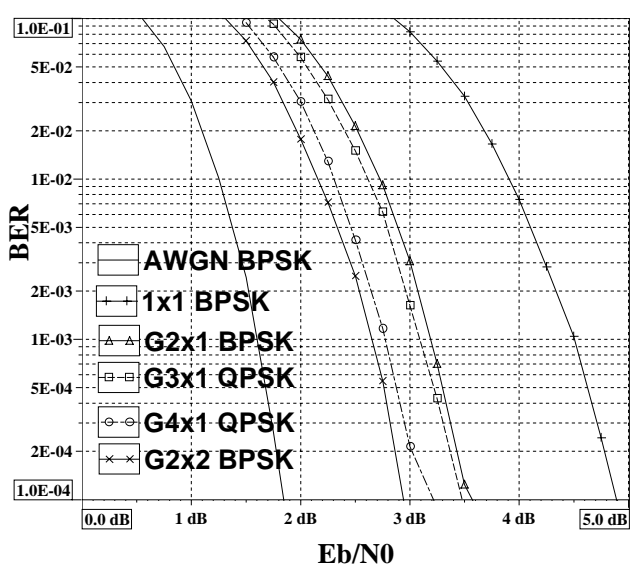

Fig. 8. Comparison of $\eta=0.5 \mathrm{bps} / \mathrm{Hz}$ systems with a $\frac{1}{2}$ rate Turbo Code

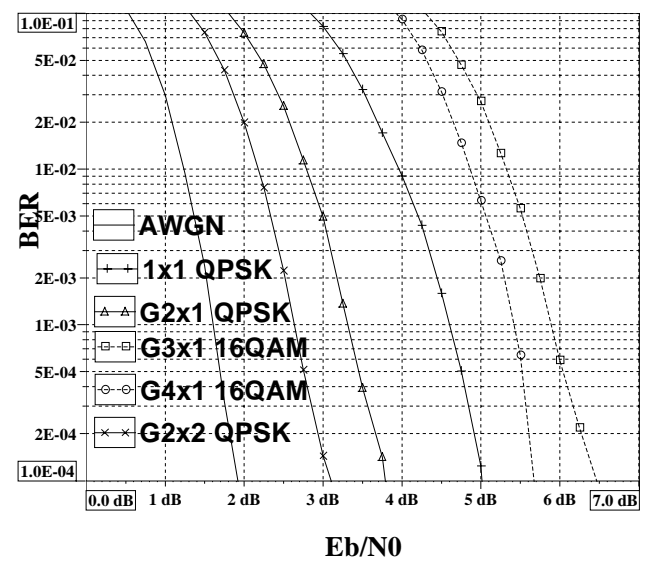

Fig. 9. Comparison of $\eta=1 \mathrm{bps} / \mathrm{Hz}$ systems with a $\frac{1}{2}$ rate Turbo Code

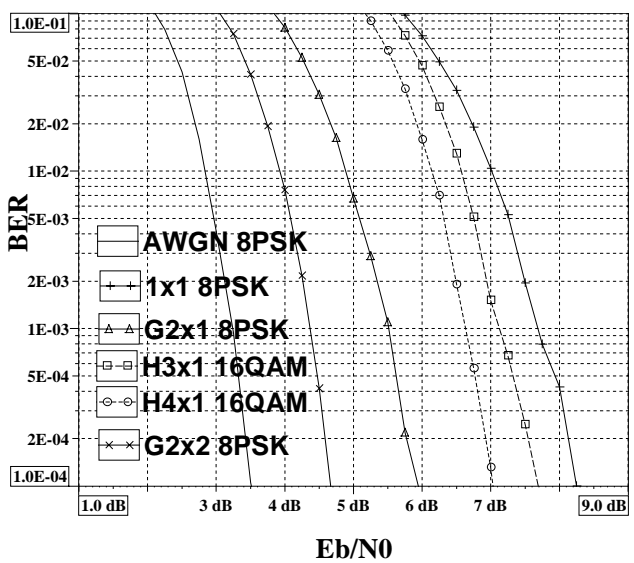

Fig. 10. Comparison of $\eta=1.5 \mathrm{bps} / \mathrm{Hz}$ systems with a $\frac{1}{2}$ rate Turbo Code

Orthogonal Designs, IEEE Transactions on Information Theory, Vol. 45, No. 5, July 1999, pp. 1456-1467.

5] G. J. Foschini, Layered space-time architecture for wireless communication in a fading environment when using multi-element antennas, Bell Labs Technical Journal, Vol. 1, No. 2, 1996, pp. 41-59.

[6] J.-M. Auffray, J.-F. Hélard, Performance of multicarrier CDMA technique combined with space-time block coding over Rayleigh channel, IEEE ISSSTA 2002, Prague, Tch. Republic, September.

[7] C. Berrou, M. Jézéquel, Non binary convolutional codes for turbo-coding, Electronic Letters, vol. 35, N. 1, January 1999, pp 39-45.

[8] IST MATRICE project, web site http://www.ist-matrice.org. 